\title{
CONTRAST ENHANCEMENT IN DIRECT-PROJECTED AUGMENTED REALITY
}

\author{
Hanhoon Park, Moon-Hyun Lee, Sang-Jun Kim, and Jong-Il Park \\ Division of Electrical and Computer Engineering \\ Hanyang University, Seoul, South Korea \\ hanuni@mr.hanyang.ac.kr,fly4moon@teramail.com, markjun@empal.com, \\ jipark@hanyang.ac.kr
}

\begin{abstract}
In direct-projected augmented reality, use of projector makes it possible to utilize 3-D real and large objects as displays and frees from discomforts incidental to wearing a device such as HMD. However, the resulting augmentation usually has poor depth resolution due to projectors with low contrast. In direct-projected augmented reality, the radiometric compensation, which is originally employed to recover the color properties of the projector input image in direct-projected augmented reality, seems to be another cause of decreasing the final contrast of the augmentation. In this paper, a contrast enhancement method is proposed that combines a hue- and saturation- preserving histogram equalization with the radiometric compensation in directprojected augmented reality. The method guarantees that the radiometrically compensated color is maintained while the brightness (or intensity) contrast is enhanced. Experimental results demonstrate the validity of our method.
\end{abstract}

\section{INTRODUCTION}

Projectors can display images with very high spatial resolution. Moreover, they are getting cheaper and their size is getting smaller. These advances led the projectors to become ubiquitous. Recently, projection display technology has gained significant attention and has been employed for a variety of applications including the construction of seamless high resolution display [1], immersive 3-D virtual environment generation [2], and synthesis of interesting visual effects or useful information on 3-D objects (directprojected augmented reality) $[3,4,6]$. However, projectors usually still suffer from low contrast and this limitation is a cause of seriously reducing the visibility of projection.

Especially, in direct-projected augmented reality, the visibility reduction by low contrast may become more serious because poor quality of augmented image significantly decreases the sense of immersion. To improve the visibility of augmentation, the problem with color or intensity distortion has been addressed in the literature $[4,5,6]$. However, these researches concentrated only on making the augmentation look as intended. They did not consider the enhancement of the augmentation because the enhancement may imply some digress from the intended image. Actually, the radiometric compensation mechanism is another cause of decreasing the contrast of the augmentation [11].

In this paper, we focus on making only the chromaticity of the projector input image unchanged after projected onto a color-textured screen. Therefore, brightness enhancement can be employed to enhance the brightness of the augmentation and significantly improve the visibility of the augmentation while preserving the original chromaticity. Our method consists of two steps: first, the augmentation is compensated using a radiometric compensation method; next, the brightness of the augmentation is enhanced using a color histogram equalization method.

There is no method for contrast enhancement in directprojected augmented reality but some contrast preserving methods exist in the literature $[10,11]$. Majumder's method [10] aimed at matching the brightness of multiple projectors with each other while trying to maintain their original brightness based on the analysis of human contrast sensitivity. However, its goal is not to enhance but to maintain the original contrast of the projectors. Wang et al. [11] employed a contrast compression method for preserving enough brightness and contrast in the radiometrically compensated image. Their method is not an enhancement method, either.

This paper is organized as follows. In Section 2, we explain what decreases the contrast of a projector input image in a direct projected augmented reality system. In Section 3, our method for contrast enhancement of directprojected augmented reality is explained. Experimental results are shown in Section 4 and conclusion is drawn in Section 5.

\section{CONTRAST DECREASE IN A DIRECT- PROJECTED AUGMENTED REALITY SYSTEM}

In a direct projected augmented reality system, the original contrast of a projector input image is decreased in a camera image as

$$
C_{C O N}=y\left(x\left(I_{C O N}\right)\right)
$$


where

$C_{C O N}$ : contrast of a camera image

$I_{C O N}:$ contrast of a projector input image

$x(\cdot)$ : contrast decrease function of a projector

$y(\cdot)$ : contrast decrease function of a camera.

The concept of radiometric compensation [5] is likely to include the recovery of the original contrast of the projector input image. However, the original contrast cannot be completely recovered due to the limited dynamic range of a projector. Actually, the contrast decrease is more intensified than recovered by the previous radiometric compensation methods. A radiometric compensation method intentionally decreases the contrast of the projector input image [11]. So, Eq. (1) becomes

$$
\hat{C}_{\text {CON }}=z\left(C_{\text {CON }}\right) \text {. }
$$

Here, $\hat{C}_{C O N}$ is the contrast of a radiometrically compensated camera image and $z(\cdot)$ is a contrast decrease function by radiometric compensation. Therefore, another method is required for resolving this contrast decrease problem.

\section{METHOD}

\subsection{Color Histogram Equalization}

Histogram equalization is one of the techniques for image enhancement for gray scale images. The generalization of this technique to color images is not straight-forward. Unlike gray scale image, there are some factors in color images such as hue and saturation which need to be properly taken care of for enhancement. For the purpose of enhancing a color image, it is to be seen that hue and saturation should not change for any pixel. If hue is changed than the color gets changed, thereby distorting the image. Saturation change sometimes degrades the quality of the image since it leads to very large saturation values that are not present in the natural scenes.

There are some color histogram equalization methods in the literature $[7,8,9]$. Among them, we use a hue-preserving color histogram equalization method [7] and modify the method to be also saturation-preserving.

\subsubsection{Hue-preserving color histogram equalization}

A simple way for hue-preserving color histogram equalization is to equalize only the luminance/saturation or both the components in the color spaces such as HIS, YIQ. However, this sometimes leads to gamut problem when the processed data is again transformed back to RGB space [7].

To avoid the gamut problem, a color histogram equalization method based on two operations, scaling and shifting, which are hue-preserving was proposed [7]. A transformation which is a combination of scaling and shifting is written as

$$
\begin{gathered}
r^{\prime}=\alpha r+\beta, \quad g^{\prime}=\alpha g+\beta, \quad b^{\prime}=\alpha b+\beta \\
\text { where } \alpha=f(r+g+b) /(r+g+b), \\
\beta=g(r+g+b) /(r+g+b) .
\end{gathered}
$$

Here, $f$ and $g$ are nonlinear transformation used in contrast enhancement for gray scale images such as histogram equalization. To reduce the complexity of the transformation, $\beta$ is taken to be zero. Then, the transformation would become

$$
r^{\prime}=\alpha r, \quad g^{\prime}=\alpha g, \quad b^{\prime}=\alpha b .
$$

In such a case that the value of $\alpha$ is greater than $1, r^{\prime}, g^{\prime}$, $b^{\prime}$ may exceed 255 and thus resulting in gamut problem. So, when the value of $\alpha$ is greater than 1 , the $r, g, b$ are transformed into CMY space and processed in the CMY space [7].

\subsubsection{Both hue- and saturation- preserving color histogram equalization}

The color equalization method explained in the previous section always decreases the saturation when the value of $\alpha$ is greater than 1, which is not always desirable. Saturation change sometimes degrades the quality of the image since it leads to very large saturation values that are not present in the natural scenes. To make the transformation of Eq. (4) saturation-preserving, normalization is employed as

$$
\begin{aligned}
& r^{\prime \prime}=r^{\prime} / m_{-} \text {val, } \\
& g^{\prime \prime}=g^{\prime} / m_{-} \text {val, } \\
& b^{\prime \prime}=b^{\prime} / m_{-} \text {val } \\
& \text { where } m_{\text {_val }}=\max \left(r^{\prime}, g^{\prime}, b^{\prime}\right) .
\end{aligned}
$$

The process in CMY color space is not required any more.

\subsection{Radiometric Compensation in Direct-Projected Augmented Reality}

The color of projection is dependent on that of the projection surface. In other words, if the color of the surface is not pure white, the projection is modulated by the color of the surface. Radiometric compensation is a technique that makes the color of projection look unchanged by adjusting the color of the projection in advance when the projection surface has colorful texture. Letting $\mathbf{I}$ be the projector input image, the projected image $\mathbf{I}_{\mathbf{P}}$ is acquired by projector response function $f$ as

$$
I_{P}=f(I) .
$$

The projected image is observed by a camera. The radiometric model of the pipeline from the projection color to the camera input radiance color is defined as

$$
R=V I_{P}+F
$$


$I_{P}:$ projected image

$F$ : ambient light

The measured camera image $\mathbf{C}$ is acquired by camera response function $g$ as

$$
C=g(R) .
$$

Once the unknown parameters are estimated, the projector input image $\hat{I}$ is compensated by $f^{-1}$ in advance such that

$$
\hat{I}=f^{-1}\left(I_{P}\right) \text {. }
$$

For detailed explanation of radiometric compensation, refer to $[4,5]$.

\subsection{Contrast Enhancement of Direct-Projected Augmented Reality}

Radiometric compensation can make the augmented projection look as intended. However, the original contrast cannot be recovered as explained in Section 2. So, the hueand saturation- preserving histogram equalization method is combined with the radiometric compensation method.

The results of radiometric compensation provide the relationship between the color of the camera image and that of the projector input image. From Eq. (6)-(9), the radiometircally compensated camera image becomes

$$
\hat{C}=g(V \cdot f(\hat{I})+F) \text {. }
$$

Let $\hat{C}_{e q}$ be the image which results from applying the histogram equalization method to $\hat{C}$. Then, to obtain the camera image $\hat{C}_{e q}$, the projector input image should be

$$
\hat{I}_{e q}=f^{-1}\left[V^{-1}\left\{g^{-1}\left(\hat{C}_{e q}\right)-F\right\}\right] .
$$

Thus, the color change of the projector input image which is required for contrast enhancement can be computed from the color change of the camera image by histogram equalization.

The procedure of our method is as follows.

i. Radiometric compensation using Eq. (9).

ii. Projecting the radiometrically compensated input image $(\hat{I})$.

iii. Capturing the projection using a camera.

iv. Applying the color histogram equalization to the camera image and compute the color change of the camera image $(\Delta C)$.

v. The color change of the projector input image $(\Delta I)$ is computed using Eq. (11).

vi. Projecting the radiometrically compensated and contrast-enhanced input image $\left(\hat{I}_{e q}=\hat{I}+\Delta I\right)$.

\section{EXPERIMENTAL RESULTS AND DISCUSSION}

Figure 1 shows our experimental setup. A projector (SONY VPL-CX6) and a camera (PointGrey Dragonfly) were used in our experiments. The images were at a resolution of 1024 by 768 pixels.

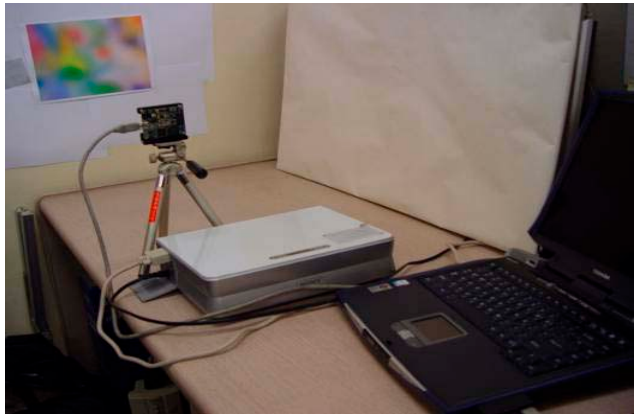

Figure 1. Experimental setup.

Figure 2 shows the results of applying the radiometric compensation and contrast enhancement method to a true color image and a gray-tone image. The contrast of the projected images without any processing is lower than that of the original images. And the radiometrically compensated images have lower contrast. The contrast enhanced images have the highest contrast and the best visibility. It means that the method contributes to the enhancement of the original contrast of the projector input image. After applying the contrast enhancement, the change of histogram is shown in Fig. 3. The method guarantees that the color calibrated by the radiometric compensation method is almost undistorted but the brightness contrast is significantly enhanced.

\section{CONCLUSION}

We proposed a contrast enhancement method for directprojected augmented reality systems that combined color histogram equalization with radiometric compensation. The method adjusted the color of the augmentation to be same as the color of the projector input image and adjusted the brightness of the augmentation to enhance brightnesscontrast. The enhancement greatly improved the visibility of augmentation.

Acknowledgement: This study was supported by a grant(02-PJ3-PG6-EV04-0003) of Ministry of Health and Welfare, Republic of Korea.

\section{REFERENCES}

[1] R. Surati, Scalable Self-Calibrating Display Technology for Seamless Large-Scale Displays, PhD thesis, MIT, 1999.

[2] R. Raskar, et al., "Multi-Projector Displays Using CameraBased Registration," Proc. of IEEE Visualization, 1999.

[3] R. Raskar, et al., "Shader Lamps: Animating Real Objects with Image-Based Illumination," Proc. of Eurographics Workshop on Rendering Techniques, pp.89-102, 2001.

[4] H. Park, M.-H. Lee, S.-J. Kim, and J.-I. Park, "SurfaceIndependent Direct-Projected Augmented Reality," Proc. of ACCV'06, pp.892-901, Jan. 2006. 


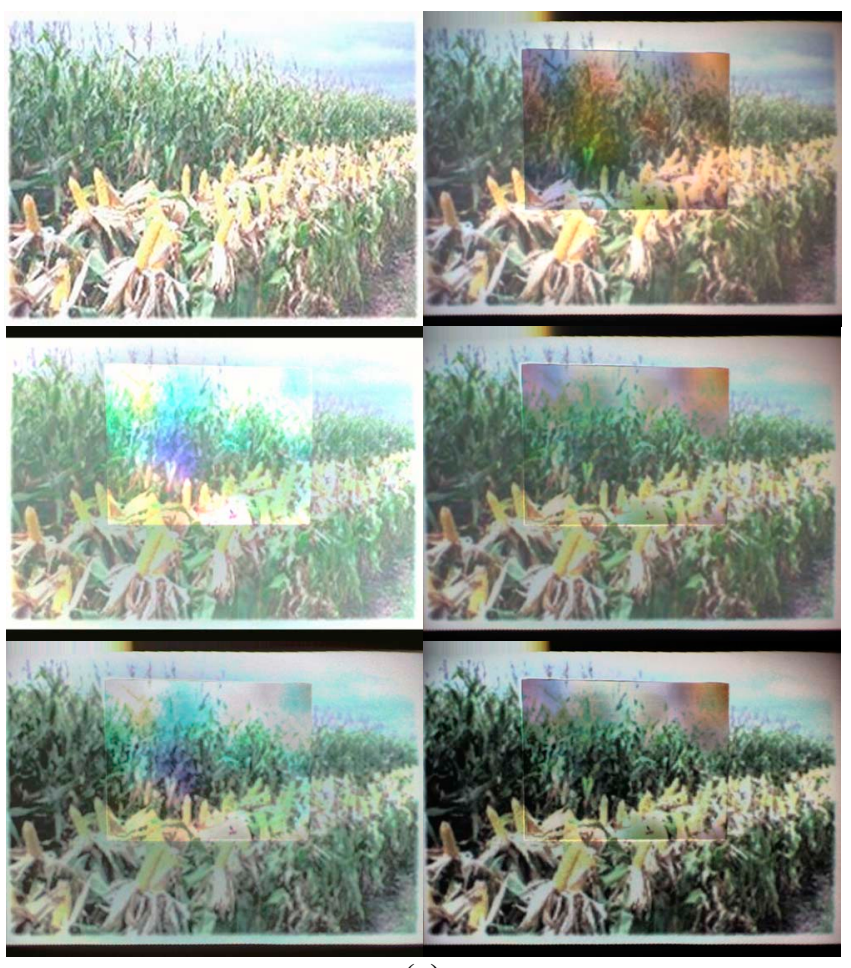

(a)

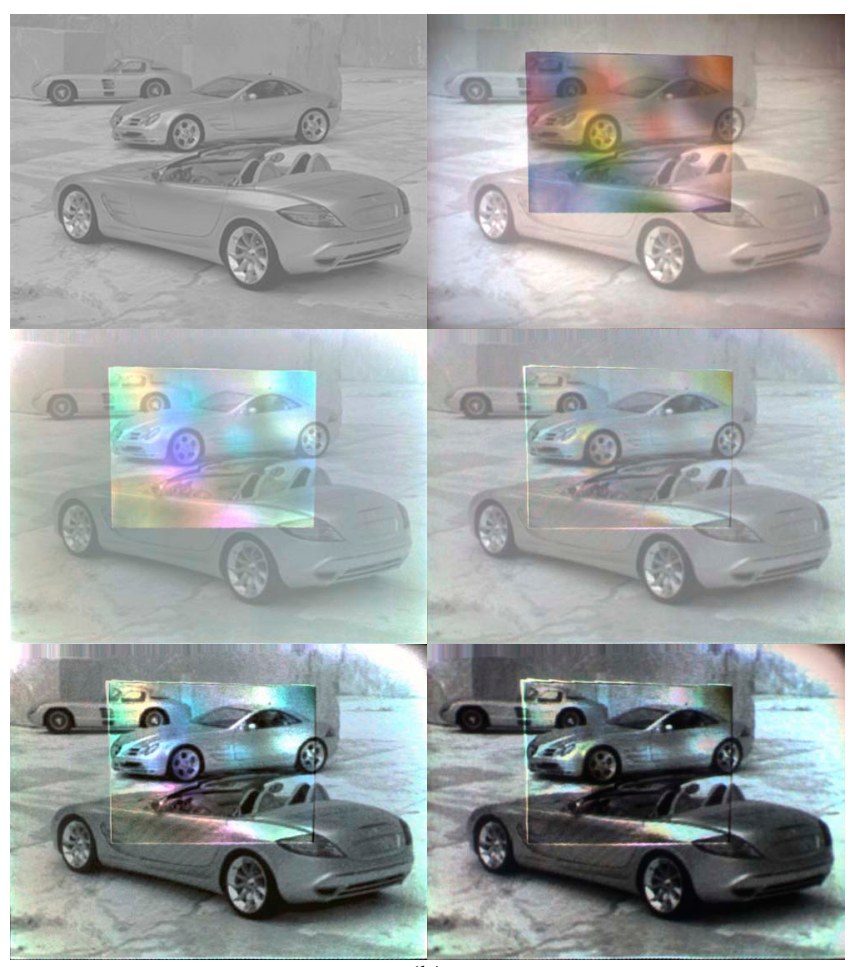

(b)

Figure 2. Radiometric compensation and contrast enhancement. (a) true color image, (b) gray-tone image. Top-left: original projector input image, top-right: image projected onto textured screen without doing anything, midleft: modified projector input image for radiometric compensation, mid-right: radiometrically compensated projection image, bottom-left: modified projector input image for contrast enhancement, bottom right: radiometrically compensated and contrast enhanced projection image. The effect of the screen texture was lessened by the radiometric compensation method but the resulting contrast became lower.

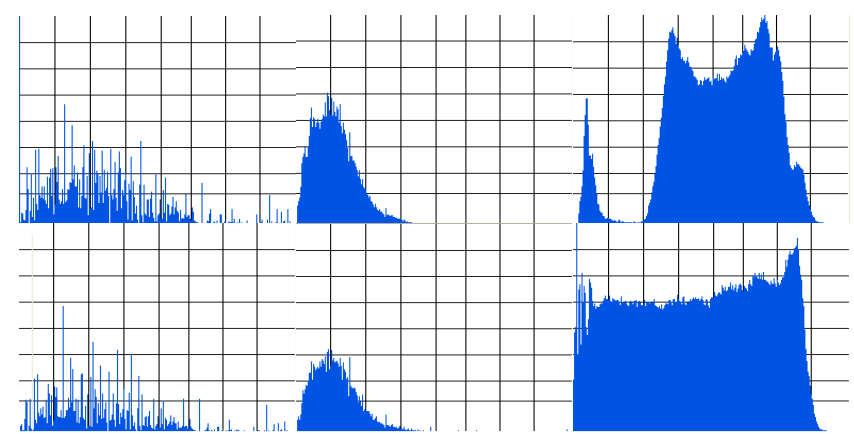

Figure 3. Hue-, saturation-, value-histogram of the mid-right image and bottom-right image in Fig. 2(a). The color is almost undistorted but the brightness (or intensity) contrast is largely enhanced.

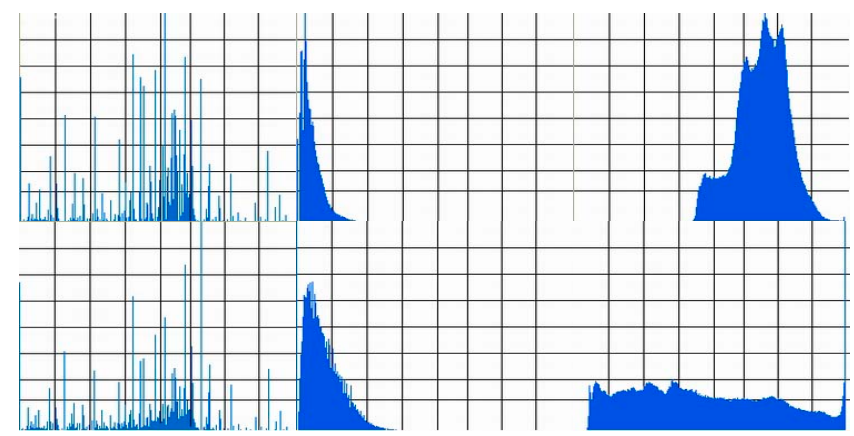

Figure 4. Hue-, saturation-, value-histogram of the mid-right image and bottom-right image in Fig. 2(b). The color is almost undistorted but the brightness (or intensity) contrast is largely enhanced.

[5] M.D. Grossberg, et al., "Making One Object Look Like Another: Controlling Appearance Using a Projector-Camera System," Proc. of CVPR'04, 2004.

[6] O. Bimber, et al., "Superimposing Pictorial Artwork with Projected Imagery," IEEE Multimedia, pp.16-26, 2005.

[7] S.K. Naik and C.A. Murthy, "Hue-Preserving Color Image Enhancement Without Gamut Problem," IEEE Transactions on Image Processing, vol.12, no.12, pp.1591-, 1598, 2003.

[8] E. Pichon, et al., "Color Histogram Equalization though Mesh Deformation,” Proc. of ICIP’03, pp.117-120, 2003.

[9] I.M. Bockstein, "Color Equalization Method and Its Application to Color Image Processing," Journal of Optical Society of America A, vol.3, no.5, pp.735-737, 1986.

[10] A. Majumder, "Contrast Enhancement of Multi-Displays Using Human Contrast Sensitivity,” Proc. of CVPR'05, 2005.

[11] D. Wang, et al., "Radiometric Compensation in a ProjectorCamera System Based on the Properties of Human Vision System," Proc. of PROCAM'05, 2005. 